\title{
New Physical Formulation of the Thermionic Emission Current at the Heterojunction Interface
}

\author{
Kow-Ming Chang, Member, IEEE, Jung-Yu Tsai, Student Member, IEEE, \\ and Chun-Yen Chang, Fellow, IEEE
}

\begin{abstract}
The thermionic emission current for electrons across the heterointerface is classically modeled as the difference between two opposing electron fluxes. Here we have developed a new consistent physical model, which includes the carrier degeneracy and nonideal behavior effects, for thermionic emission current at the heterojunction interface. It is shown that the thermionic emission current at the heterojunction interface can be expressed in a simple closed-form formalism which gives the relations among the average directional thermal velocity of electrons, the conduction band discontinuity, and the carrier activities at both sides of the interface. We also discuss the conditions under which the thermionic emission occurs at the heterointerface.
\end{abstract}

\section{INTRODUCTION}

$I_{\text {diser }}$ T IS generally recognized from metal-semiconductor diode theory [1] that the thermionic emission phenomenon at the heterojunction interface is one of the important transport mechanisms for the flow of electron (or hole) current across a heterojunction. The classical thermionic model often neglects the effective mass, degeneracy, and density-of-state effects on both sides of the interface as given by (24) below. In addition, the conventional thermionic emission current is usually modeled as the difference between two opposing electron (or hole) fluxes that do not relate anything about the carriers behavior at (or near) the heterojunction interface.

In this paper, we present a new, simple, but general self-consistent thermionic emission formulation for the carrier transport across a heterojunction interface from an irreversible thermodynamic point of view [2], which includes the nonideal behavior of the carriers around the interface. The correlation between the thermionic emission current and the activities of the carriers shows that the thermodynamic parameters are important to the transport mechanisms that determine the thermionic current. The result presented here gives insight into the thermionic transport problems of the carriers across a heterointerface. We also compare the interesting results

Manuscript received December 4, 1992. This work was supported by the ROC National Science Council, Engineering Division under Grant NSC 81-0404-E009-54

The authors are with the Department of Electronics Engineering and the Institute of Electronics, National Chiao Tung University, Hsin-chu, Taiwan, Republic of China.

IEEE Log Number 9210269. with those that appeared in several preceding papers. Finally, the necessary conditions of the thermionic current transport mechanism across the heterointerface are also discussed.

\section{THEORY}

Consider a one-dimensional (n)GaAlAs/(p)GaAs abrupt emitter-base heterojunction with ohmic contacts at $x=0$ on the n-side and $x=w$ on the p-side, and with the heterointerface at $x_{j}$ as shown in Fig. 1(a). The energy band diagram of the junction near forward-biased condition is illustrated in Fig. 1(b), where $E_{l}$ is the local vacuum level, $\chi$ is the electron affinity, $E_{G}$ is the bandgap energy, $E_{C}$ is the bottom of the conduction band, $E_{V}$ is the top of the valence band, $E_{F n}$ is the electron quasiFermi level, $E_{F p}$ is the hole quasi-Fermi level, $\Delta E_{C}\left(=\chi_{B}\right.$ $-\chi_{E}$ ) is the conduction band discontinuity, $\Delta E_{V}$ is the valence band discontinuity, and $x_{n}$ and $x_{p}$ are the spacecharge-region edges on the $\mathrm{n}$ and $\mathrm{p}$ sides, respectively.

Here we will focus our analysis on the consistent derivation of the thermionic emission current across the heterojunction interface. We follow the approach in [3] and [4] and include the Fermi-Dirac statistics. The thermionic emission current across the heterointerface can be expressed as

$$
\begin{aligned}
& J_{n}=-A^{*} T^{2} e^{-\phi_{B} / k T} e^{-V_{d}^{p} / k T} \\
& \cdot\left[\exp \left(\frac{-q \phi_{n}\left(x_{j}^{-}\right)}{k T}\right)-\exp \left(\frac{-q \phi_{n}\left(x_{j}^{+}\right)}{k T}\right)\right]
\end{aligned}
$$

where $A^{*}\left(=4 q \pi k^{2} m^{*}\left(x_{j}^{+}\right) / h^{3}\right.$ for the special case of spherical bands) is the Rechardson's constant and $m^{*}\left(x_{j}^{+}\right)$ is the effective mass of the electron in the base region. As pointed out in [3], the $A^{*}$ and $m^{*}$ values depend on the band structure of the heterojunction. $\phi_{B}=E_{G B}-V_{p}-$ $V_{b i}^{p}+\chi_{B}-\chi_{E}$, where $V_{p}=E_{F}^{p}(w)-E_{V B}(w)$ and $V_{b i}^{p}$ is the build-in potential across the $\mathrm{p}$-side region. $V_{a}^{p}$ represents the portion of the applied voltage that appears across the p-type junction. $\phi_{n}(x)=\left[E_{F}^{p}(w)-E_{F n}(x)\right] / q$ is the quasi-Fermi potentials for the electrons. It should be noted here that quasi-equilibrium condition can be applied at $x=w$. Thus, by convention, we have $E_{F n}(w)=$ $E_{F p}(w) \equiv E_{F}^{p}(w)$. 


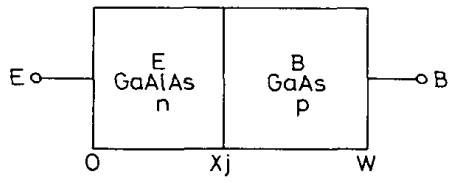

(a)

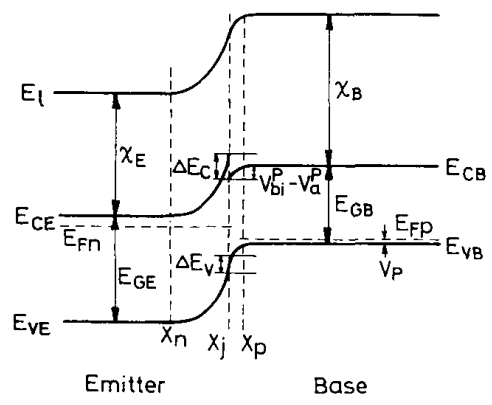

(b)

Fig. 1. (a) One-dimensional n-p emitter-base heterojunction device structure; (b) qualitative energy band diagram for (a) under forwardbiased condition, including the partial hole and electron quasi-Ferm levels, and the band discontinuities.

If we assume Fermi-Dirac statistics and write the equilibrium electron density as

$$
n_{0}(x)=N_{C} \exp \left(\frac{E_{F}-E_{C}}{k T}\right) \zeta_{C}
$$

after some algebraic manipulation, the thermionic emission current across the junction can be written as

$$
\begin{aligned}
J_{n}=\frac{-q S_{K L}^{P} n_{0}\left(x_{p 0}\right)}{\zeta_{c}\left(x_{p 0}\right)} \exp \left(\frac{q V_{j p}-\Delta E_{C}}{k T}\right) \\
\cdot\left[\exp \left(\frac{-q \phi_{n}\left(x_{j}^{-}\right)}{k T}\right)-\exp \left(\frac{-q \phi_{n}\left(x_{j}^{+}\right)}{k T}\right)\right]
\end{aligned}
$$

where $\zeta_{C}(x)\left(=F_{1 / 2}\left(\eta_{C}\right) / \exp \left(\eta_{C}\right)\right)$ is the degeneracy effect for semiconductors with parabolic bands and $F_{1 / 2}$ is the Fermi-Dirac integral of order one-half with

$$
\eta_{C}(x)=\frac{E_{F_{n}}(x)-E_{C}(x)}{k T} .
$$

$V_{j p}$ is the potential drop on the p side of the junction and is given by

$$
V_{j p}=V_{b i}^{p}-V_{a}^{p}=V\left(x_{j}\right)-V\left(x_{p}\right) .
$$

$S_{k L}\left(=\left(\left[k T / 2 \pi m^{*}\left(x_{j}^{+}\right)\right]\right)^{1 / 2}\right)$ is the average directional thermal velocity of electrons (in p-side material) moving in the direction perpendicular to the interface. The subscript zero in (3) denotes equilibrium. Also, we assume that $n_{0}(W)=n_{0}\left(x_{p 0}\right)$. This assumption is good for the uniformly heavily doped base band structure [5]. Note that the Fermi-Dirac integral of order one-half $\left(F_{1 / 2}\right)$ used here implies that the standard parabolic or spherical band structure is assumed. This is not the general situation for the case of high doping [5]. It is also noted that FermiDirac statistics for the electrons on both sides of the heterointerface are used in the derivation of (1). In addition, the electron temperatures of each side of the interface are assumed to be equal. This implies that the hot-electron injection effects (e.g., ballistic transport and velocity overshoot effects) can be neglected.

The electron concentration in an isothermal compositionally nonuniform degenerate semiconductor can be related to its activity coefficient in the following general form [2]:

$$
n(x)=\frac{n_{i}(w)}{\gamma_{n}(x)} \exp \left[\frac{q\left(V(x)-\phi_{n}(x)\right)}{k T}\right]
$$

and

$$
\gamma_{n}(x)=\exp \left(\frac{-A \Delta E_{g}}{k T}\right)
$$

where $A$ is the effective asymmetry factor and $\Delta E_{g}$ is the effective bandgap shrinkage which accounts for the actual bandgap narrowing effect, the density-of-state effects, and the influence of Fermi-Dirac statistics. Here we choose $x=w$ as the reference position for the electrostatic potential. Parameters $\Delta E_{g}$ and $A$ are defined as

$$
\begin{aligned}
\Delta E_{g}=\left[E_{G}(w)-E_{G}(x)\right]+ & k T \ln \left[\frac{N_{C}(x) N_{V}(x)}{N_{C}(w) N_{V}(w)}\right] \\
+k T & \ln \left[\zeta_{C}(x) \zeta_{V}(x)\right]
\end{aligned}
$$

and

$$
A=\frac{\left[(\chi(x)-\chi(w))+k T \ln \left(\frac{N_{C}(x)}{N_{C}(w)}\right)+k T \ln \left(\zeta_{C}(x)\right)\right]}{\Delta E_{g}} .
$$

The activity coefficient here accounts for the nonideal behavior of the carriers due to heavy doping effects and nonuniform band structure. This coefficient is a function of electron affinity, bandgap, doping density, and the density of states that vary with position. It is important to mention here that, for $A=1 / 2$ (symmetric case), (6) is reduced to the conventional degenerate result as [6]

$$
n(x)=n_{i e}(x) \exp \left[\frac{q\left(V(x)-\phi_{n}(x)\right)}{k T}\right]
$$

where $n_{i e}(x)$ is an effective intrinsic carrier concentration as defined in [2]. Generally, this condition (i.e., $A=1 / 2$ ) is not the case for the real systems.

The electron concentration at $x_{j}^{-}$is obtained by putting $x=x_{j}^{-}$in (6) as

$$
\begin{aligned}
& n\left(x_{j}^{-}\right)=n_{i}(w) \\
& \cdot \exp \left[\frac{q\left(V\left(x_{j}^{-}\right)-\phi_{n}\left(x_{j}^{-}\right)\right)+A \Delta E_{g}\left(x_{j}^{-}\right)}{k T}\right] .
\end{aligned}
$$


The intrinsic carrier concentration $n_{i}(w)$ in (11) can be related to the equilibrium electron concentration at $x=$ $x_{p 0}$ in (6) as

$$
n_{i}(w)=n_{0}\left(x_{p 0}\right) \exp \left[\frac{-q V^{0}\left(x_{p 0}\right)-A \Delta E_{g}^{0}\left(x_{p 0}\right)}{k T}\right] .
$$

Substituting (12) into (11), followed by some rearrangement, gives

$$
\begin{aligned}
n_{0}\left(x_{p 0}\right) & \exp \left(\frac{-q \phi_{n}\left(x_{j}^{-}\right)}{k T}\right) \\
= & n\left(x_{j}^{-}\right) \exp \left[\frac{q\left(V\left(x_{p 0}\right)-V\left(x_{j}^{-}\right)\right)}{k T}\right] \\
& \cdot \exp \left[\frac{A \Delta E_{g}\left(x_{p 0}\right)-A \Delta E_{g}\left(x_{j}^{-}\right)}{k T}\right] .
\end{aligned}
$$

In a similar manner, we have the results for the case at $x=x_{j}^{+}$as

$$
\begin{aligned}
n_{0}\left(x_{p 0}\right) & \exp \left(\frac{-q \phi_{n}\left(x_{j}^{+}\right)}{k T}\right) \\
= & n\left(x_{j}^{+}\right) \exp \left[\frac{q\left(V\left(x_{p 0}\right)-V\left(x_{j}^{+}\right)\right)}{k T}\right] \\
& \cdot \exp \left[\frac{A \Delta E_{g}\left(x_{p 0}\right)-A \Delta E_{g}\left(x_{j}^{+}\right)}{k T}\right] .
\end{aligned}
$$

Furthermore, using the following equations

$$
\begin{gathered}
A \Delta E_{g}\left(x_{p 0}\right)=k T \ln \left[\zeta_{C}\left(x_{p 0}\right)\right] \\
A \Delta E_{g}\left(x_{j}^{+}\right)=k T \ln \left[\zeta_{C}\left(x_{j}^{+}\right)\right] \\
A \Delta E_{g}\left(x_{j}^{-}\right)=-\Delta E_{C}+k T \ln \left[\frac{N_{C}\left(x_{j}^{-}\right)}{N_{C}(w)}\right] \\
+k T \ln \left[\zeta_{C}\left(x_{j}^{-}\right)\right] \\
V\left(x_{j}^{-}\right)=V\left(x_{j}^{+}\right)=V\left(x_{j}\right)
\end{gathered}
$$

and substituting (5), (13), and (14) into (3), followed by some algebraic manipulations, gives

$$
J_{n}=-q S_{k L}\left[\frac{n\left(x_{j}^{-}\right)}{\zeta_{C}\left(x_{j}^{-}\right)} \frac{N_{C}^{p}}{N_{C}^{n}}-\frac{n\left(x_{j}^{+}\right)}{\zeta_{C}\left(x_{j}^{+}\right)} e^{-\Delta E_{C} / k T}\right] .
$$

Here we assume that $N_{C}\left(x_{j}^{-}\right)=N_{C}^{n}$ and $N_{C}(w)=N_{C}^{p}$. It should be mentioned here that simple closed-form selfconsistent current-voltage characteristics for the carrier transport by drift and diffusion across the space-charge region (conventional model) along with the thermionic emission across the heterointerface (as presented here) can be readily connected and obtained from [2] for the abrupt heterojunction diodes and bipolar transistors with heavily doped concentrations and nonuniform band structure under one-dimensional analysis. The results will be published elsewhere in the future.
Equation (19) can be further rearranged to get the new simple formulation of the thermionic emission current at the heterojunction interface. Two cases will be studied here. First, if we move $e^{-\Delta E_{s} / k T}$ in the second term of (19) out of the parentheses and use (7), (16) and (17), then we obtain

$$
\begin{aligned}
J_{n} & =-q S_{K L}^{p} e^{-\Delta E_{C} / k T}\left[n\left(x_{j}^{-}\right) \gamma_{n}\left(x_{j}^{-}\right)-n\left(x_{j}^{+}\right) \gamma_{n}\left(x_{j}^{+}\right)\right] \\
& =-q S_{K L}^{p} e^{-\Delta E_{C} / k T}\left[a_{n}\left(x_{j}^{-}\right)-a_{n}\left(x_{j}^{+}\right)\right]
\end{aligned}
$$

where $a_{n}$ is the activity of the electron and is the product of concentration and activity coefficient. Equation (20) is a new, general, and simple formulation that relates the thermionic emission current to the difference of the electron activity on both sides of the interface. In addition, it is clear from (20) that the magnitude of the $J_{n}$ is also determined by the average of the directional thermal velocity $S_{k L}^{p}$ and the conduction band discontinuity $\Delta E_{C}$. Secondly, if we move $N_{C}^{p} / N_{C}^{n} \zeta_{C}\left(x_{j}^{-}\right)$in the first term of (19) out of the parentheses and again use (7), (16), and (17), then we have

$$
J_{n}=\frac{-q S_{K L}^{n}}{\zeta_{C}\left(x_{j}^{-}\right)}\left[\frac{m^{*}\left(x_{j}^{+}\right)}{m^{*}\left(x_{j}^{-}\right)}\right]\left[n\left(x_{j}^{-}\right)-n\left(x_{j}^{+}\right) \Gamma\left(x_{j}\right)\right]
$$

where

$$
S_{K L}^{n}=\left[\frac{k T}{2 \pi m^{*}\left(x_{j}^{-}\right)}\right]^{1 / 2}
$$

and

$$
\begin{aligned}
\Gamma\left(x_{j}\right) \equiv & \frac{\gamma_{n}\left(x_{j}^{+}\right)}{\gamma_{n}\left(x_{j}^{-}\right)} \\
= & \exp \left\{-\left[\Delta E_{C}+k T \ln \left[\frac{N_{C}\left(x_{j}^{+}\right)}{N_{C}\left(x_{j}^{-}\right)}\right]\right.\right. \\
& \left.\left.+k T \ln \left[\frac{\zeta_{C}\left(x_{j}^{+}\right)}{\zeta_{C}\left(x_{j}^{-}\right)}\right]\right] / k T\right\}
\end{aligned}
$$

represent the relative nonideal behavior of electrons at the junction interface. Equation (21) represents the general form of the thermionic emission current for electrons across the heterointerface modeled as the difference between two opposing electron fluxes. If we neglect the effective mass, degeneracy, and density-of-states effects on both sides of the interface, then (21) is reduced to

$$
J_{n}=-q S_{K L}^{n}\left[n\left(x_{j}^{-}\right)-n\left(x_{j}^{+}\right) e^{-\Delta E_{C} / k T}\right] .
$$

Equation (24) gives the simplified classical result as that given by Grinberg et al. [7]. Apparently, the results presented above can be used to study the band discontinuity.

A conditions of the thermionic current transport mechanism across the heterojunction interface can be readily obtained. For the case of (1) and (2), we have

$$
\phi_{n}\left(x_{j}^{-}\right) \neq \phi_{n}\left(x_{j}^{+}\right)
$$


or

$$
E_{F n}\left(x_{j}^{-}\right) \neq E_{F n}\left(x_{j}^{+}\right) .
$$

From (20) or (21), the condition must satisfy either

$$
a_{n}\left(x_{j}^{-}\right) \neq a_{n}\left(x_{j}^{+}\right)
$$

or

$$
n\left(x_{j}^{-}\right) \neq n\left(x_{j}^{+}\right) \Gamma\left(x_{j}\right) .
$$

\section{CONCLUSION}

This paper reports on some interesting, general, and new formulations of the thermionic emission current across the heterojunction interface. The new results account for the nonideal behavior of electrons on both sides of the interface. In addition, this new physical model is both thermodynamically consistent and consistent with the electronic transport equations. Finally, it should be pointed out that the results presented here are quite general and can also be readily extended to a consistent solution for the case of hole thermionic transport across the valence band heterointerface.

\section{ACKNOWLEDGMENT}

The authors are indebted to anonymous reviewers for a careful reading and criticism of the manuscript.

\section{REFERENCES}

[1] S. M. Sze, Physics of Semiconductor Devices, 2nd ed. New York: Wiley, 1981.

[2] K. M. Chang, "A consistent model for carrier transport in heavily doped semiconductor devices," Semicond. Sci. Technol., vol. 3, pp. $766-772,1988$.

[3] S. J. Fonash, "Band structure and photocurrent collection in crystalline and polycrystalline p-n heterojunction solar cells," Solid-State Electron., vol. 22, pp. 907-910, 1979.

[4] S. J. Fonash, "General formulation of the current-voltage characteristics of a p-n heterojunction solar cell," J. Appl. Phys., vol. 51, pp. $2115-2118,1980$

[5] K. M. Chang and J. Y. Tsai, "Two new generalized equations for minority-carrier transport in bipolar transistors with heavily doped base and non-uniform band structure," Semicond. Sci. Technol. vol. 7, pp. 799-803, 1992.

[6] S. Selberherr, Analysis and Simulation of Semiconductor Devices. Vienna: Springer-Verlag, 1984

[7] A. A. Grinberg, M. S. Shur, R. J. Fischer, and H. Morkoç, "An investigation of the effect of graded layers and tunneling on the performance of AlGaAs / GaAs heterojunction bipolar transistors," IEEE Trans. Electron Devices, vol. ED-31, pp. 1758-1764, 1984. 